\title{
Nonlinear optical properties of CdTe nanocrystals synthesized by a green room temperature solution method
}

\author{
M. H. Majles Ara $\cdot$ Z. Moslemi $\cdot$ H. Naderi \\ A. Mihandoost $\cdot$ A. Daneshfar $\cdot$ R. Sahraei
}

Received: 19 July 2014 / Accepted: 3 February 2015 / Published online: 13 February 2015

(c) The Author(s) 2015. This article is published with open access at Springerlink.com

\begin{abstract}
The CdTe nanocrystals were successfully synthesized using a facile room temperature method with non-toxic and environmentally friendly capping agent. The obtained CdTe nanocrystals were characterized by UV-Vis absorption spectroscopy, transmission electron microscopy, and z-scan technique. A photoluminescence peak with full width at half maximum of around $15 \mathrm{~nm}$ could be obtained, which indicates a homogeneous and narrow size distribution for the CdTe nanocrystals. The magnitude and sign of the third-order nonlinear refractive index $\left(n_{2}\right)$ and nonlinear absorption coefficient $(\beta)$ of the CdTe nanocrystals were determined by using of the closed- and open-aperture $\mathrm{z}$-scan at different intensities, respectively. The negative sign of $n_{2}$ indicated that there is a self-defocusing effect in the sample. The nonlinear refractive index, $n_{2}$, and the nonlinear absorption coefficient, $\beta$, were in order of $10^{-7} \mathrm{~cm}^{2} \mathrm{~W}^{-1}$ and $10^{-2}-10^{-3} \mathrm{cmW}^{-1}$, respectively.
\end{abstract}

\section{Introduction}

In the last decade, the optical properties of semiconductor nanocrystals have been widely investigated due to their possible application in fields of optoelectronic, photo-catalysis, and biological labeling [1]. The semiconductor nanocrystals or quantum dots have different application ranging from optoelectronic devices [2-5] to cancer treatments [6].

\footnotetext{
M. H. Majles Ara $(\bowtie) \cdot$ Z. Moslemi $\cdot$ H. Naderi

Photonics Laboratory, Department of Physics, Kharazmi

University, 49 Mofatteh Ave., Tehran, Iran

e-mail:majlesara@gmail.com; majlesara@tmu.ac.ir

A. Mihandoost · A. Daneshfar · R. Sahraei

Department of Chemistry, University of Ilam, P.O. Box:

65315-516, Ilam, Iran
}

These materials exhibit quantum confinement effects when their sizes are comparable with the diameter of the bulk exciton, leading to split the continuum of electronic energy levels into discrete states with an effective band gap blue shifted from that of the bulk [7-9].

There are two different synthetic methods for producing $\mathrm{CdTe}$ nanostructures, the organometallic route and synthesis in an aqueous solution [10-13]. The organometallic method usually requires hard conditions such as high temperature, toxic reagents and solvents, and a rigorous process for protecting synthesized nanostructures from oxidation, while the aqueous synthesis method requires mild conditions and is a simpler one [14, 15]. Compared with organometallic route, aqueous synthesis has many advantages such as biocompatibility, water solubility, environmental friendliness, and low cost [16]. The aqueous synthetic approach allows the resulting nanostructures to be tuned in terms of their size, shape, and structure on the nanometer scale [17]. The capping agent is one of the most important factors in the synthesis of nanostructures. Some of the commonly used methods for surface passivation include protection by self-assembled monolayers, the most popular being thiol-functionalized organics; encapsulation in the water pools of reverse microemulsions; and dispersion in polymer matrixes [18]. The thiol-functionalized organic compounds are highly reactive chemically and pose potential environmental and biological risks. It is well known that capping ligands attached on the nanostructure surface have strong effects on the size, morphology, and compatibility of nanostructures with different chemical mediums [19]. Therefore, it is of great importance to have a proper selection of appropriate capping ligands, which would lead to the desired particle structures. Sodium citrate is one of the most common agents used in the synthesis of metallic and semiconductor nanostructures because 
it can act as a reducing reagent and/or as a capping agent [20-23]. This capping agent provides the nanoparticles with sufficient electrostatic stabilization to avoid agglomeration for long periods of time. It also plays a key role in the growth process and the photoluminescence quantum yields of aqueous CdTe nanocrystals [12, 13]. In this work, a green room temperature method using the sodium citrate as a capping agent for environmentally friendly synthesis of CdTe nanocrystals is introduced.

Also, optical properties of nanocrystals depend on electronic structure, chemical compound, and nanocrystals sizes. Recently, nanometer-sized semiconductor materials have been intensively investigated since they exhibit large optical nonlinearity and fast response time due to their novel electronic structures originated from quantum confinement and interfacial effect [24]. Therefore, we investigated the nonlinear optical properties of CdTe nanocrystals that prepared by chemical method, such as the sign and magnitude of both the nonlinear absorption and refractive index and linear absorption. The refractive index of these materials, which is partly dependent upon the input intensity, has either a focusing or a defocusing effect on the incident laser beam. This nonlinear refractive index is a highly important parameter in designing optical devices [25]. Different techniques for measuring nonlinear refractive index of the material have been employed, each technique having its advantages and disadvantages. These techniques include nonlinear interferometry [26], degenerate four-wave mixing [27], nearly degenerate three-wave mixing [28], ellipse rotation [29], and beam distortion measurements [30]. Here we utilize a technique for this purpose which is known as z-scan [25, 31]. The z-scan technique, based on principles of spatial beam distortion, provides a simple sensitive tool for determining both the nonlinear refractive index and the nonlinear absorption coefficient.

\section{Experimental}

All chemicals including cadmium perchlorate hexahydrate $\left(\mathrm{Cd}\left(\mathrm{ClO}_{4}\right)_{2} \cdot 6 \mathrm{H}_{2} \mathrm{O}\right)$, tellurium (Te) powder, tri-sodium citrate dehydrate $\left(\mathrm{C}_{6} \mathrm{H}_{5} \mathrm{O}_{7} \mathrm{Na}_{3} \cdot 2 \mathrm{H}_{2} \mathrm{O}\right)$, sodium borohydride $\left(\mathrm{NaBH}_{4}\right), \mathrm{NaOH}$, and $\mathrm{HCl}$ with the highest purity available were purchased from Merck Co. (Darmstadt, Germany) and used without further purifications.

Sodium borohydride $(2.0 \mathrm{mmol})$ was reacted with tellurium powder $(0.5 \mathrm{mmol})$ in a $50.0-\mathrm{mL}$ round-bottom flask containing $10.0-\mathrm{mL}$ ultra-pure water and under $\mathrm{N}_{2}$ atmosphere to produce sodium hydrogen telluride (NaHTe). The reaction flask was kept in an ice bath at $5.0{ }^{\circ} \mathrm{C}$. After $24.0 \mathrm{~h}$, sodium tetraborate was produced as white precipitate at the bottom of the flask and the color of the solution changed to a faint pink. The synthesis of CdTe nanocrystals was based on the reaction of cadmium perchlorate hexahydrate $\left(\mathrm{Cd}\left(\mathrm{ClO}_{4}\right)_{2} \cdot 6 \mathrm{H}_{2} \mathrm{O}\right)$ with sodium hydrogen telluride $(\mathrm{NaHTe})$. All reactions were carried out in oxygenfree water under nitrogen. In a typical synthesis, $3 \mathrm{~mL}$ of $\mathrm{Cd}\left(\mathrm{ClO}_{4}\right)_{2} \cdot 6 \mathrm{H}_{2} \mathrm{O}$ solution $\left(0.05 \mathrm{molL}^{-1}\right)$ was added under stirring to a $20 \mathrm{~mL}$ aqueous solution of sodium citrate $(0.1 \mathrm{wt} \%)$ at room temperature. The solution is placed in a three-necked flask fitted with a septum and valves and is deaerated by $\mathrm{N}_{2}$ bubbling for $30 \mathrm{~min}$. Under stirring, $1 \mathrm{~mL}$ of freshly prepared NaHTe solution $\left(0.05 \mathrm{molL}^{-1}\right)$ was injected into the solution together with a slow nitrogen flow for $30 \mathrm{~min}$. At this stage, the color of the solution changed from clear to transparent light brown. This coloration change is believed to be due to the nucleation and growth of citrate stabilized CdTe nanocrystals.

Absorption measurements were carried out on a PerkinElmer Lambda 25 spectrophotometer using a quartz cell with an optical path of $1 \mathrm{~cm}$. The morphology and size distribution of the CdTe nanocrystals were determined by a Hitachi H-800 transmission electron microscope (TEM) at an operating voltage of $200 \mathrm{kV}$. Samples TEM were prepared by putting a drop of the colloidal solution on a copper grid coated with a thin amorphous carbon film. The excess solvent was removed using a filter paper and letting the solvent evaporate at room temperature. X-ray diffraction (XRD) pattern was recorded with an automated Philips X'Pert X-ray diffractometer with $\mathrm{Cu} \mathrm{K} \alpha$ radiation $(40 \mathrm{kV}$ and $30 \mathrm{~mA}$ ) for $2 \theta$ values over $10-70^{\circ}$. The investigation of the nonlinear optical characteristics of the CdTe nanocrystals suspensions was carried out using the $\mathrm{CW} \mathrm{He}-\mathrm{Ne}$ laser and the transmission z-scan technique.

The z-scan technique is one of the best ways for the nonlinear absorption and refraction coefficient measurement. The basis of this method is the changes in a $\mathrm{TEM}_{00}$ Gaussian beam profile that traveling in the $\mathrm{z}$ direction, when the sample moves back or forth along the $z$-axis near the focal point [32].

Now consider a sample with third-order nonlinearity where refractive index is

$n(I)=n_{0}+\Delta n=n_{0}+n_{2} I$

where $n_{0}$ is the linear refractive index, $I$ denotes the intensity of incident light at the entrance plane of sample, and $\Delta n$ is the refractive index change.

When the sample was positioned near the beam waist, the transmittance was measured with a power meter behind an aperture. The sample was moved along the direction of beam propagation, the $\mathrm{z}$ direction, and the transmittance versus sample position gives information about the nonlinearities. The beam transmitted from a lens or a sample changes in the phase but does not influence its Gaussian shape. The laser radiation was focused by an 8 -cm focal length lens.

This method involves two experimental setups: First, a closed-aperture setup is used for calculating the sign and 
Fig. 1 Systematical setup of closed-aperture z-scan technique

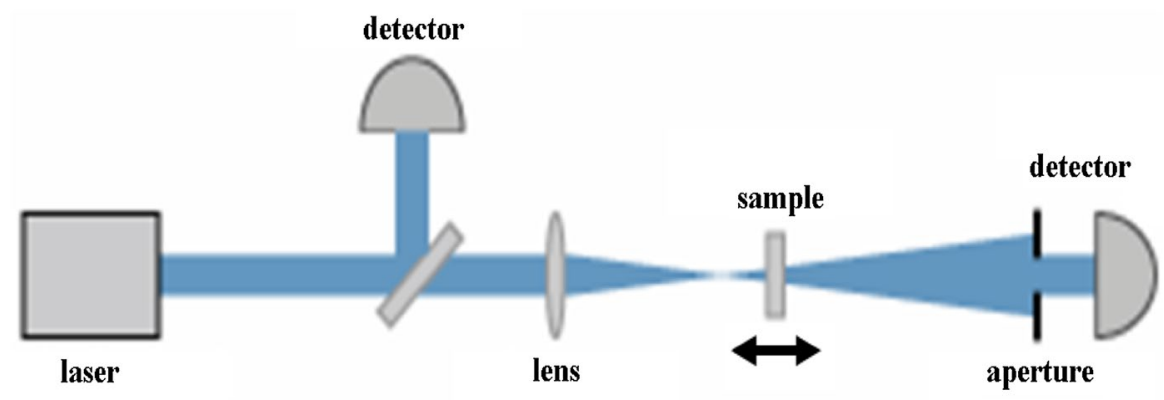

magnitude of nonlinear refraction index $\left(n_{2}\right)$, and second, an open-aperture setup yields the nonlinear absorption coefficient $(\beta)$. The experimental setup for z-scan is schematically shown in Fig. 1.

The value of aperture linear transmission, $S$, is 0.13 . The nonlinear refraction index $\left(n_{2}\right)$ can be calculated from the peak-to-valley height $\left(\Delta T_{p-v}\right)$ of the normalized smoothed curve of experimental points as follows [33]:

$\Delta T_{p-v}=f \Delta \Phi_{0} \quad$ for $\Delta \Phi_{0}<\pi$

where $\Delta T_{p-v}$ is the difference between the normalized peak transmittance and valley transmittance, $f=0.406(1-S)^{0.25}$ is an experimental constant, and $\Delta \Phi_{0}$ is the plane distortion. $\Delta \Phi_{0}$ relates to $n_{2}$ through the following expression:

$\Delta \Phi_{0}=\frac{2 \pi L_{\mathrm{eff}} n_{2} I_{0}}{\lambda}$

where $L_{\mathrm{eff}}=\left(1-e^{-\alpha L}\right) / \alpha$ ( $\alpha$ is the linear absorption $)$ is the effective thickness of the sample, $I_{0}=2 P_{0} / \pi w_{0}^{2}$ is the incident illumination intensity at focal point, $P_{0}$ is the laser power, $w_{0}=32 \mu \mathrm{m}$, and $L=1 \mathrm{~mm}$ is the length of the sample.

The second setup, which is open aperture, yields the nonlinear absorption coefficient $(\beta)$ was measured without an aperture $(s=1$, the total transmittance without the aperture). The normalized transmittance under open-aperture conditions is given by

$T_{\text {norm }}(z)=\frac{\operatorname{Ln}\left(1+q_{0}(z, t)\right)}{q_{0}(z, t)}$

where $q_{0}(z, t)=\beta I_{0} L_{\text {eff }} /\left(1+z^{2} / z_{0}^{2}\right), z_{0}=k \omega_{0 / 2}^{2}$ is the diffraction length of the beam, $\beta$ is the nonlinear absorption, and $k=2 \pi / \lambda$ is the wave vector [34].

\section{Results and discussion}

Figure 2a shows UV-Vis absorption spectrum of the citrate-capped CdTe nanocrystals recorded at room temperature. The CdTe nanocrystals solution show a

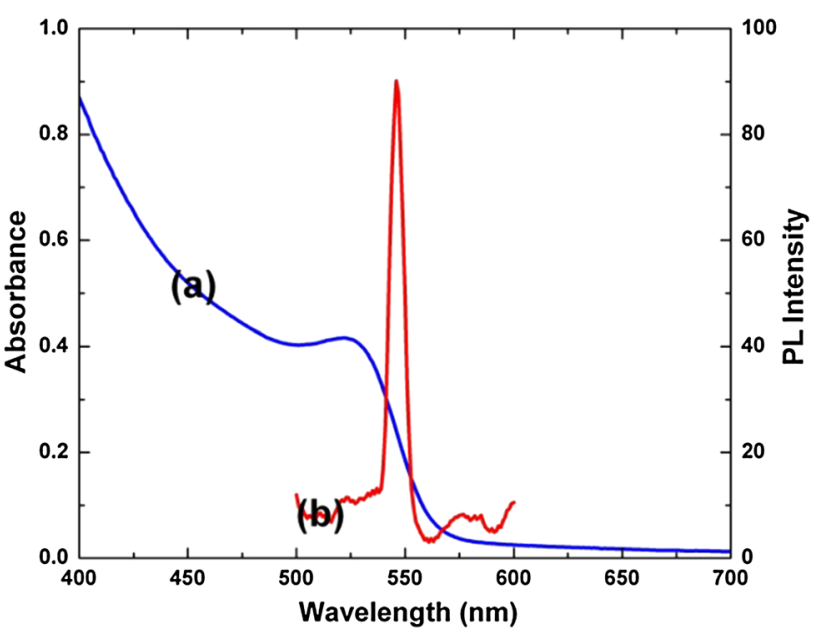

Fig. 2 UV-Vis absorption (a) and PL (b) spectra of the citratecapped CdTe nanocrystals taken as prepared

well-resolved absorption maximum of the first electronic transition indicating a sufficiently narrow size distribution of the CdTe nanocrystals, which shifts to the shorter wavelengths with decreasing size of the nanocrystals as a consequence of the quantum confinement. The relative sharpness of the absorption edge is attributed to the formation of the CdTe nanocrystals with a narrow size distribution. So there is a blue shift of at least $250 \mathrm{~nm}$, $0.75 \mathrm{eV}$ in energy, in comparison with that of the bulk $\mathrm{CdTe}$ at $800 \mathrm{~nm}(1.5 \mathrm{eV})$. This blue shift in the absorption edge is most probably due to the quantum size effect as expected from the nano-sized nature of the $\mathrm{CdTe}$ nanocrystals [2].

The photoluminescence (PL) measurements were taken to study the optical properties of the CdTe nanocrystals. The PL measurements were carried out at room temperature, and the excitation wavelength was $400 \mathrm{~nm}$. As shown in Fig. 2b, the PL spectrum is dominated by a single large peak centered in $550 \mathrm{~nm}$. Also, the full width at half maximum of this PL spectrum exhibited a very slight broadening of a few nanometer indicating a sufficiently narrow size distribution of the CdTe nanocrystals. 
A typical TEM image of the CdTe nanocrystals is displayed in Fig. 3a. In general, the CdTe nanocrystals are isotropic in shape. As we anticipated from the absorption data, the characteristic spherical CdTe nanocrystals are observed with a relatively narrow particle size distribution $(2-11 \mathrm{~nm}$ range). A histogram of the CdTe nanocrystals size distribution is also presented in Fig. 3b. The average nanocrystals diameter observed is $5.3 \mathrm{~nm}$ (standard deviation, $\mathrm{SD}=2.6 \mathrm{~nm}$ ), and more than $93 \%$ of the CdTe nanocrystals are in the size range from 2 to $9 \mathrm{~nm}$, indicating a relatively monodispersity of the CdTe nanocrystals formed in the system.

XRD pattern of the CdTe nanocrystals was shown in Fig. 3c. The standard XRD pattern for CdTe (Joint Committee for Powder Diffraction Standards, JCPDS card No. 10-0207) is given at the bottom of Fig. 3c. The three broad peaks observed in the diffractogram at around $23.7^{\circ}, 39.4^{\circ}$, and $46.6^{\circ}$ reveal a cubic lattice structure of CdTe. These peaks can be assigned to the planes (111), (220), and (311), respectively, of the cubic phase [12]. From the full width at half maximum of diffraction peaks, the average size of the CdTe nanocrystals was calculated using the Debye-Scherrer equation [35], as around $5 \mathrm{~nm}$.

We calculate the linear absorption $\alpha=1.03\left(\mathrm{~cm}^{-1}\right)$ by measuring output power with a sample as a function of the power without the sample in low powers.

Also, the experimental results show that the effective length $\left(L_{\text {eff }}\right)$ is obtained as $0.95 \mathrm{~mm}$ by the linear absorption. Figure 4 shows the curve of normalized transmittance of closed-aperture z-scan in different intensities of focal point (with $80-\mathrm{cm}$ focal length). The curve is characterized by a pre-focal peak followed by a post-focal valley, indicating that the nonlinear refraction of the samples is negative and belongs to self-defocusing nonlinearity [36, 37]. Because of the small value of $\beta$ in our experiments, the closed-aperture $\mathrm{z}$-scan curve obtained is relatively symmetrical in shape [38]. We obtained the quantity of $\Delta T_{p-v}$ by Fig. 4 . Then the nonlinear refractive index $\left(n_{2}\right)$ of the CdTe nanocrystals is calculated by Eqs. (2) and (3) and is reported in different intensities in Table 1.

Figure 5 shows the open-aperture data, where $s=1$. The solid curve is fitted by using Eq. (4) together with the experimental parameters. The normalized transmittance is a function of the sample position $\mathrm{z}$. The sign of $\beta$ is positive because we have valley in open figures. This result suggests that this nonlinearity is mainly due to two-photon absorption process [39]. The nonlinear absorption coefficient $(\beta)$ was calculated from Eq. (4). Calculated values of nonlinear absorption coefficient at different intensities are shown in Table 1.
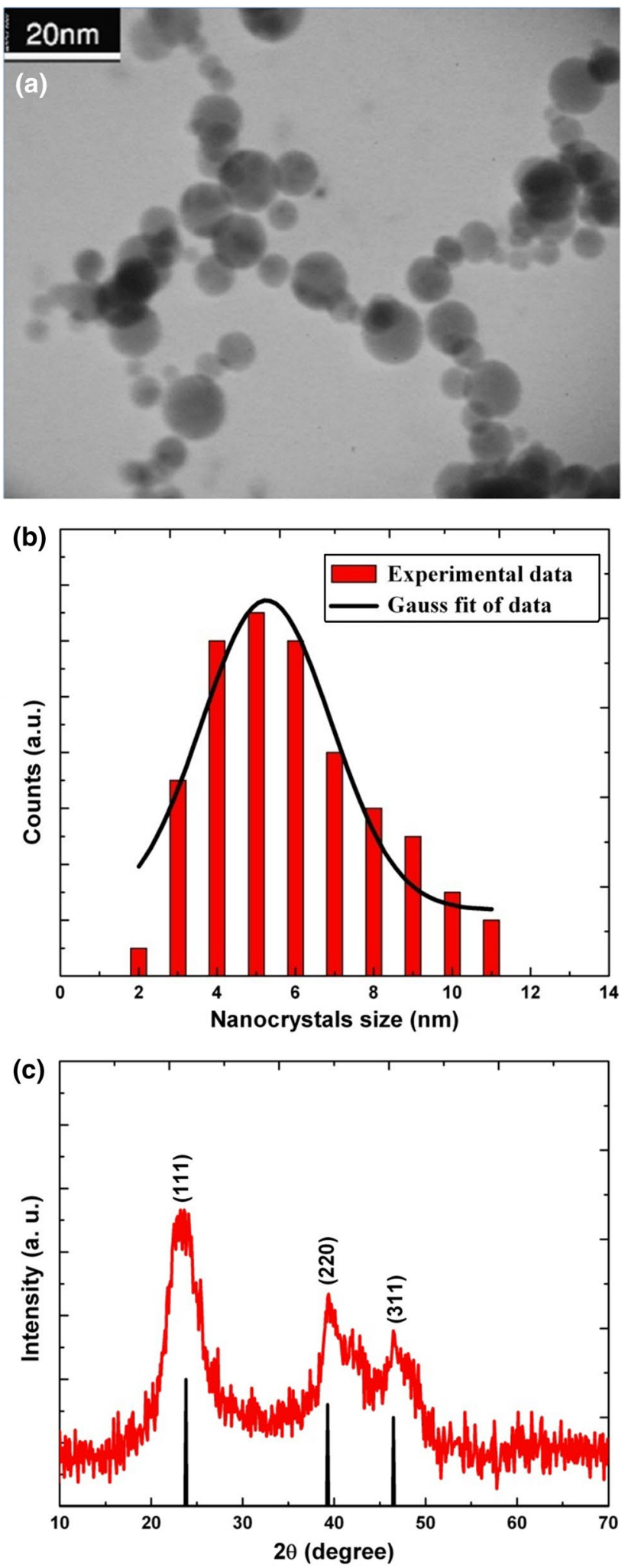

Fig. 3 a Typical TEM image, b histogram of the particles size distribution, and $\mathbf{c}$ the XRD pattern of the citrate-capped CdTe nanocrystals 


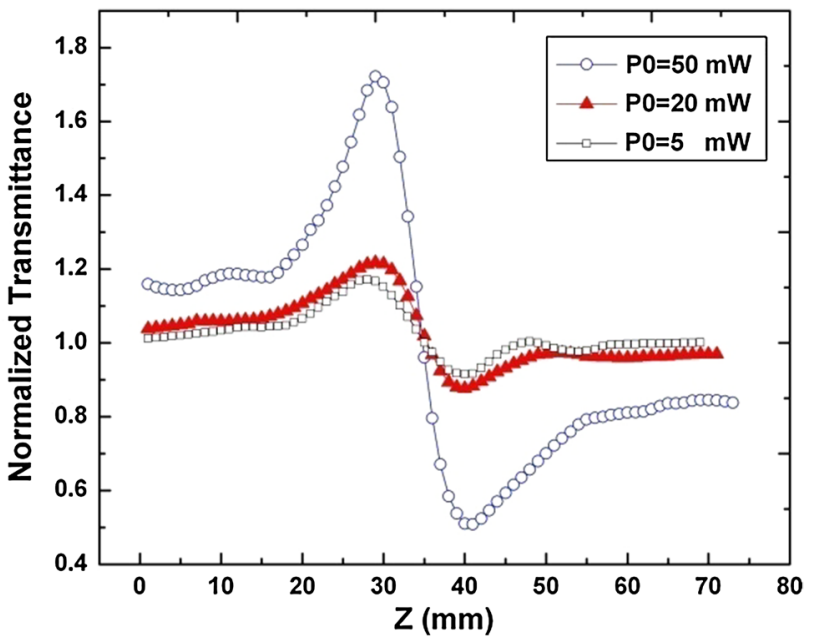

Fig. 4 Closed-aperture z-scan experimental curves of the CdTe nanocrystals at three incident intensities; the solid line is best fit to experimental points

Table 1 Calculated values of nonlinear absorption coefficient and nonlinear refractive index at three incident intensities

\begin{tabular}{llll}
\hline $\mathrm{P}_{0}(\mathrm{~mW})$ & $\alpha\left(\mathrm{cm}^{-1}\right)$ & $\mathrm{n}_{2}\left(\mathrm{~cm}^{2} / \mathrm{W}\right)$ & $\beta(\mathrm{cm} / \mathrm{W})$ \\
\hline 5 & 1.03 & $1.93 \times 10^{-7}$ & $2.7 \times 10^{-3}$ \\
20 & 1.03 & $2.05 \times 10^{-7}$ & $1.2 \times 10^{-2}$ \\
50 & 1.03 & $2.58 \times 10^{-7}$ & $1.4 \times 10^{-2}$ \\
\hline
\end{tabular}

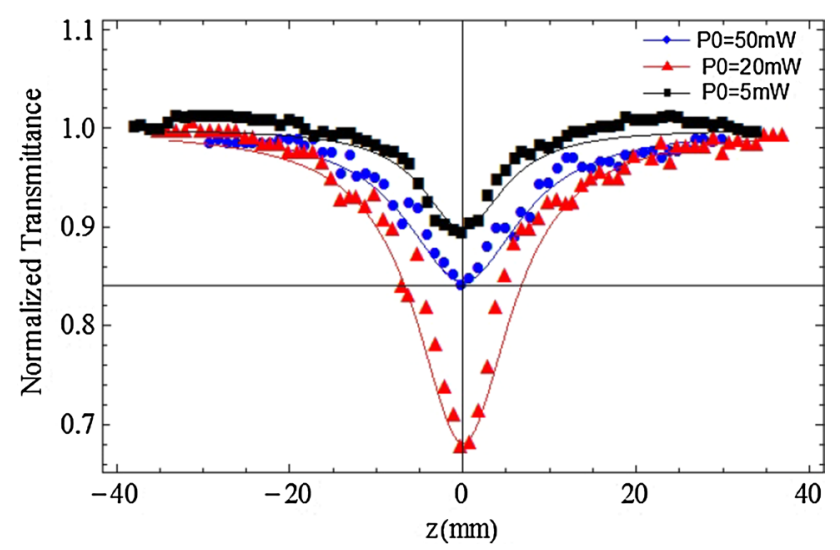

Fig. 5 Open-aperture z-scan experimental curves of the CdTe nanocrystals at three incident intensities; the solid line is best fit to experimental points

\section{Conclusion}

In summary, the CdTe nanocrystals were prepared by a green room temperature solution method. Structural measurements through XRD analysis demonstrated that the synthesized CdTe nanocrystals have a cubic structure. The size of these nanocrystals was found to be in the range of 2-11 $\mathrm{nm}$ as analyzed using transmission electron micrographs. The third-order nonlinear optical properties of the CdTe nanocrystals were determined by using the single beam z-scan technique, and according to the obtained data from closed-aperture $\mathrm{z}$-scan, $n_{2}$ has negative sign. The negative sign of $n_{2}$ indicates that this material exhibits self-defocusing optical nonlinearity. According to the open-aperture z-scan results, the sign of $\beta$ is positive. This indicates that two-photon absorption is the dominant absorption effect in these intensities.

Open Access This article is distributed under the terms of the Creative Commons Attribution License which permits any use, distribution, and reproduction in any medium, provided the original author(s) and the source are credited.

\section{References}

1. R. Comparelli, F. Zezza, M. Striccoli, M.L. Curri, R. Tommasi, A. Agostiano, Mater. Sci. Eng. C 23, 1083 (2003)

2. N. Gaponik, D.V. Talapin, A.L. Rogach, K. Hoppe, E.V. Shevchenko, A. Kornowski, H. Weller, A. Eychmulller, J. Phys. Chem. B 106, 7177 (2002)

3. M.C. Schlamp, X. Peng, A.P. Alivisatos, J. Appl. Phys. 82, 5837 (1997)

4. H. Mattoussi, L.H. Radzilowski, B.O. Dabbousi, E.L. Thomas, M.G. Bawendi, M.F. Rubner, J. Appl. Phys. 83, 7965 (1998)

5. M.Y. Gao, C. Lesser, S. Kirstein, H. Mohwald, A.L. Rogach, H. Weller. J. Appl. Phys. 87, 2297 (2000)

6. E.A. Forssen, Z.A. Tokes, J. Cancer Res. 43, 546 (1983)

7. K.S. Babu, C. Vijayan, P. Haridoss, Mater. Res. Bull. 42, 1251 (2007)

8. Y.F. Liu, J.S. Yu, J. Colloid Interface Sci. 333, 690 (2009)

9. J.M. Klostranec, W.C.W. Chan, J. Adv. Mater. 18, 1953 (2006)

10. W.W. Yu, L. Qu, W. Guo, X. Peng, Chem. Mater. 15, 2854 (2003)

11. H. Liu, J.S. Owen, A.P. Alivisatos, J. Am. Chem. Soc. 129, 305 (2007)

12. C.L. Wang, H. Zhang, S.H. Xu, N. Lv, Y. Liu, M.J. Li, H.Z. Sun, J.H. Zhang, B. Yang, J. Phys. Chem. C 113, 827 (2009)

13. Y. Wang, Z. Tang, M.A. Correa-Duarte, I. Pastoriza-Santos, M. Giersig, N.A. Kotov, L.M. Liz-Marzan, J. Phys. Chem. B 108, 15461-15469 (2004)

14. Z.A. Peng, X. Peng, J. Am. Chem. Soc. 124, 3343 (2002)

15. J.V. Williams, C.N. Adams, N.A. Kotov, P.E. Savage, Ind. Eng. Chem. Res. 46, 4358 (2007)

16. Z. Gu, L. Zou, Z. Fang, W. Zhu, X. Zhong, Nanotechnology 19, 135604 (2008)

17. A.L. Rogach, T. Franzl, T.A. Klar, J. Feldmann, N. Gaponik, V. Lesnyak, A. Shavel, A. Eychmüller, Y.P. Rakovich, J.F. Donegan, J. Phys. Chem. C 111, 14628 (2007)

18. A. Ullman, Chem. Rev. 96, 1533 (1996)

19. A.C.S. Samia, J.A. Schlueter, J.S. Jiang, S.D. Bader, C.J. Qin, X.M. Lin, Chem. Mater. 18, 5203 (2006)

20. X. Ji, X. Song, J. Li, Y. Bai, W. Yang, X. Peng, J. Am. Chem. Soc. 129, 13939 (2007)

21. J. Zeng, Y. Zheng, M. Rycenga, J. Tao, Z.Y. Li, Q. Zhang, Y. Zhu, Y. Xia, J. Am. Chem. Soc. 132, 8552 (2010)

22. P.P. Ingole, R.M. Abhyankar, B.L.V. Prasad, S.K. Haram, Mater. Sci. Eng. B 168, 60 (2010) 
23. R. Sahraei, M. Nosrati, A. Daneshfar, S. Abbasi, M.H. Majles Ara, Mater. Lett. 68, 153 (2012)

24. T. Ning, P. Gao, W. Wang, H. Lu, W. Fu, Y. Zhou, D. Zhang, X. Bai, E. Wang, G. Yang, Opt. Mater. 31, 931 (2009)

25. M. Sheik-bahae, A.A. Said, T.H. Wei, D.J. Hagan, E.W. Van Stryland, IEEE J. Quantum Elect. 26, 760 (1990)

26. M.J. Moran, C.Y. She, R.L. Carman, IEEE J. Quantum Elect. 11, 259 (1975)

27. S.R. Friberg, P.W. Smith, IEEE J. Quantum Elect. 11, 259 (1975)

28. R. Adair, L.L. Chase, S.A. Payne, J. Opt. Soc. Am. B 4, 875 (1987)

29. A. Owyoung, IEEE J. Quantum Elect. 9, 1064 (1973)

30. W.E. Williams, M.J. Soileau, E.W. Van Stryland, Opt. Commun. 50, 256 (1984)

31. M. Sheik-Bahae, A.A. Said, E.W. Van Stryland, Opt. Lett. 14, 955 (1989)

32. M.H. Majles Ara, E. Koushki, S. Salmani, S.H. Mousavi, Opt. Commun. 278, 418 (2007)
33. M.H. Majles Ara, Z. Dehghani, S.S. Iranizad, Int. J. Mod. Phys. B 22, 3165 (2008)

34. M.C. Nascimento, M.A.R.C. Alencar, S. Chavez-Cerda, M.G.A. da Silva, M.R. Meneghetti, J.M. Hickmann, J. Opt. A Pure Appl. Opt. 8, 947 (2006)

35. A. Goudarzi, G.M. Aval, R. Sahraei, H. Ahmadpoor, Thin Solid Films 516, 4953 (2008)

36. M.H. Majles Ara, Z. Dehghani, R. Sahraei, G. Nabiyouni, Opt. Commun. 283, 1650 (2010)

37. J.W.T. Spinks, R.J. Woods, An Introduction to Radiation Chemistry (Wiley-Interscience, New York, 1976)

38. M.H. Majles Ara, E. Koushki, S.H. Mousavi, S. Salmani, M. Rafizadeh, A. Gharibi, Mater. Chem. Phys. 109, 320 (2008)

39. M.H. Majles Ara, Z. Dehghani, E. Saievar Iranizad, Int. J. Nanotechnol. 6, 1006 (2009) 